\title{
EFECTO DE LA ESTRATIFICACIÓN FRÍA Y LA COBERTURA PLÁSTICA EN SEMILLAS DE GULUPA (Passiflora edulis Sims.) PARA LA OBTENCIÓN DE PLÁNTULAS
}

\section{EFFECT OF STRATIFICATION AND PLASTIC COVER ON PURPLE PASSION FRUIT (Passiflora edulis Sims.) SEEDS TO OBTAIN SEEDLINGS}

\author{
Helber Enrique Balaguera', Javier Giovanni Álvarez², Julián Cárdenas³
}

\begin{abstract}
${ }^{1}$ I.A. Grupo de Investigaciones Agrícolas, Facultad de Ciencias Agropecuarias, Universidad Pedagógica y Tecnológica de Colombia. Av. Central del Norte Km 0 vía Paipa Tunja, Colombia. e-mail: enrique_balaguera@yahoo.com² Profesor asistente. Grupo de Investigaciones Agrícolas, Facultad de Ciencias Agropecuarias, Universidad Pedagógica y Tecnológica de Colombia. Av. Central del Norte Km 0 vía Paipa Tunja, Colombia. e-mail: jgalvarezh@gmail.com ${ }^{3}$ I.A. Grupo de Investigaciones Agrícolas, Facultad de Ciencias Agropecuarias, Universidad Pedagógica y Tecnológica de Colombia. Av. Central del Norte Km 0 vía Paipa Tunja, Colombia. e-mail: julianentero@gmail.com
\end{abstract}

Rev. U.D.C.A Act. \& Div. Cient. 13 (2): 89-97, 2010

\section{RESUMEN}

Debido al auge de la gulupa y a su importancia económica en Colombia, se hace necesario mejorar las técnicas de producción de este cultivo. La gulupa, se propaga, comercialmente, mediante semillas, pero la dureza e impermeabilidad de la testa origina problemas de germinación y de vigor germinativo, que complican las labores de propagación y la obtención uniforme de las plántulas. Con el fin de lograr material de buena calidad para transplante, en un diseño completamente al azar bifactorial de $4 \times 2$, se estratificaron semillas de gulupa a $4^{\circ} \mathrm{C}$, durante $0,360,720$ y $1440 \mathrm{~h}$, con cubierta plástica negra en la siembra y sin ella, durante 15 días, para un total de ocho tratamientos. La siembra, se realizó en bandejas de germinación de 72 alvéolos REF. A-BA72; el experimento tuvo una duración de 60 días y se llevó a cabo en condiciones de invernadero. La germinación, se activó solo con los tratamientos de 360 y $720 \mathrm{~h}$ de estratificación y 15 días de cubrimiento con plástico negro, durante la germinación y $360 \mathrm{~h}$ sin la puesta de plástico. Los mayores valores de porcentaje de emergencia, velocidad media de germinación y altura de planta, se observaron en el tratamiento de 360h de estratificación fría y 15 días de cubrimiento con plástico negro. Los tratamientos de frío y plástico negro son complementarios para una germinación óptima. Las semillas que permanecieron $720 \mathrm{~h}$ en estratificación con 15 días de cubrimiento de plástico, durante la germinación, presentaron el mayor tiempo medio de emergencia, pero generaron las plántulas con mayor longitud de raíz.

Palabras clave: Plástico negro, horas frío, emergencia de plántulas.

\section{SUMMARY}

Due to the rise of purple passion fruit and its economic importance in Colombia it is necessary to improve the production techniques of this crop. The purple passion fruit is propagated commercially by seeds, but its coat is very hard, with a high impermeability causing problems for germination and germination forcing, which complicates propagation and seedlings uniformity. In order to achieve good quality material for transplantation, in a completely randomized bifactorial design of $4 \times 2$, purple passion fruit seeds were stratificated at $4^{\circ} \mathrm{C}$ for $0,360,720$ and 1440 hours, covering with and without black plastic mulch at planting for 15 days, for a total of eight treatments. Seedlings were planted in trays of 72 cavities REF. A-BA72; the experiment lasted 60 days and was conducted under greenhouse conditions. Germination was activated only with the treatments of 360 and $720 \mathrm{~h}$ and 15 days of covering with black plastic and $360 \mathrm{~h}$ without black plastic. The highest germination percentage and average germination speed and plant height were observed in the treatment of $360 \mathrm{~h}$ stratification and 15 days of covering with 
black plastic. The combination of cold and black plastic is considered complementary for optimum germination. The seeds, 720 h stratificated, with 15 days covered with plastic showed the largest average emergency time, but generated seedlings with the largest main root.

Key words: Black plastic, cold time, seedlings emergency.

\section{INTRODUCCIÓN}

La gulupa (Passiflora edulis Sims.) es una fruta originaria de Brasil, Paraguay y el norte de Argentina (Morton, 1987; Nakasone $\&$ Paull, 1998) y posee un gran valor por su sabor y su acción nutricional (provitamina A, niacina, riboflavina y ácido ascórbico) (Wenkam, 1990). El fruto, se caracteriza por el color púrpura oscuro de su corteza y contiene pequeñas semillas negras comestibles, cubiertas por un arilo jugoso amarillo-naranja (Calzada, 1970).

Actualmente, en Colombia, la gulupa ocupa el tercer renglón dentro de las frutas exportadas hacia el mercado europeo, después del banano y la uchuva (Pinzón et al. 2007); a pesar de esto, los estudios sobre su fisiología aún son escasos.

Delanoy et al. (2006) y Esteves et al. (2009) concuerdan en que existen pocos registros sobre germinación en Passiflora spp; sin embargo, se ha demostrado que las semillas presentan latencia en algunas especies de esta familia como: P. edulis f. flavicarpa Deg. (Alexandre et al. 2004), P. incarnata L. (Wehtje et al. 1985), P. mollissima (Kunth) L.H. Bailey (La Rosa, 1984) y P. nitida Kunth (Passos et al. 2004). Las semillas de $P$. edulis Sims. f. flavicarpa muestran diferentes grados de latencia, registrando germinaciones en periodos que van desde los 15 hasta los 45 días (Camacaro et al. 2005).

Las semillas latentes, se hallan en un estado fisiológico, en el cual, permanecen viables, pero no pueden germinar, incluso, en condiciones ambientales favorables. Esto evita que la germinación se produzca en condiciones donde la sobrevivencia de las plántulas se vea amenazada (Schmidt, 2000).

Las semillas pueden presentar latencia al momento de ser dispersadas de la planta, latencia primaria o, desarrollarla, debido a las condiciones ambientales, latencia secundaria. Para el primer caso, puede ser, por una parte, exógena: producida por factores externos al embrión; física, puesto que muestra testa impermeable; mecánica, donde la testa es dura e impide la protrusión de la radícula; química, cuando hay inhibidores en la testa o pericarpo y, por otra, endógena: causada por factores a nivel del embrión; puede ser dividida en morfológica, ocasionada porque el embrión está poco desarrollado y fisiológica, que está relacionada con el componente hormonal (Hartmann et al. 1997).

Cuando la latencia se debe a condiciones de la testa, el letargo termina en el momento en que ésta se agrieta o debilita por acciones mecánicas o químicas (escarificación) o por efecto del ambiente (Madueño-Molina et al. 2006). Hartmann $\varepsilon$ Kester (2002) reportan que esta situación es frecuente en gran número de familias de plantas, en las que la testa y/o secciones endurecidas de otras cubiertas son impermeables, debido a que en la superficie exterior se forma una capa gruesa, revestida de sustancias cerosas y tejido esclerenquimático (Rolston, 1978).

El tiempo de almacenamiento influye sobre el poder germinativo de las semillas de maracuyá (Carlesso et al. 2008). El almacenamiento en nevera de semillas de maracuyá dulce $(P$. alata) produce mayor germinación comparado con el que se hace a temperatura ambiente, mientras que el almacenamiento en refrigerador ha demostrado ser efectivo para mantener la viabilidad de estas semillas (Alves et al. 2006).

La luz constituye un factor de gran importancia en la germinación de las semillas y en el desarrollo de las plántulas. Cada especie exterioriza una sensibilidad diferente a la luz, de tal manera, que existen semillas cuya germinación puede ser influenciada positiva o negativamente por la luz y semillas cuya germinación es indiferente a este factor (Borges $\mathcal{E}$ Rena, 1993; Zucareli et al. 2009).

A pesar de que la información sobre semillas de pasifloras ha avanzado en los últimos años, en gulupa no existen estudios sobre su germinación. Ellis et al. (1985) reportaron que las semillas latentes de Passiflora spp. requieren escarificación y temperaturas alternas $\left(20-30^{\circ} \mathrm{C}\right)$, para promover la germinación. La alternancia de temperatura puede producir cambios físicos en la cubierta de las semillas que favorecen la absorción de agua e inducen la germinación. Al respecto, Pereira $E$ Andrade (1994) encontraron que en maracuyá la alternancia de temperatura de 20 a $30^{\circ} \mathrm{C}$ generó mayor germinación respecto a temperaturas constantes $\left(20\right.$ ó $\left.30^{\circ} \mathrm{C}\right)$.

Hechenleitner et al. (2005) reportan que en P. pinnatistipula Cav. las semillas se deben remojar por $24 \mathrm{~h}$ en agua fría y sembrar inmediatamente; si fueron secadas deben ser remojadas en agua tibia ( $21 \mathrm{a} 27^{\circ} \mathrm{C}$ ), por $24 \mathrm{~h}$. Mencionan los mismos autores, que la germinación mejora al mezclar las semillas con el jugo y la pulpa del fruto fresco, ya que el ácido del jugo ayuda a romper la testa de la semilla y estimula la germinación.

Por lo anterior, el objetivo de esta investigación fue evaluar el efecto del tiempo de estratificación a $4^{\circ} \mathrm{C}$ y la cobertura plástica durante la germinación de semillas de gulupa sobre 
la emergencia y obtención de plántulas, con el fin de buscar alternativas para mejorar la producción en esta especie.

\section{MATERIALES Y MÉTODOS}

La investigación, se realizó en un invernadero de la Facultad de Ciencias Agropecuarias de la Universidad Pedagógica y Tecnológica de Colombia, sede Tunja, ubicada a $73^{\circ} 23^{\prime}$ de longitud Oeste y $5^{\circ} 32$ ' de latitud Norte y a una altitud de 2690 msnm. La temperatura promedio en el interior del invernadero fue de $16^{\circ} \mathrm{C}$ y la HR de $65 \%$.

Se utilizaron semillas de gulupa provenientes de frutos maduros cosechados en la vereda Guamal, del municipio de Miraflores (Boyacá), ubicada a aproximadamente 1700msnm; presenta una temperatura promedio de $18^{\circ} \mathrm{C}$ y humedad relativa del $80 \%$. Las semillas fueron sembradas en bandejas de 72 alvéolos de REF. A-BA72.

Se manejó un diseño completamente al azar con arreglo bifactorial de $4 \times 2$; el primer factor fue el tiempo de estratificación fría a $4^{\circ} \mathrm{C}(0,360,720$ y 1440 h) y el segundo correspondió a la puesta o no de cubierta plástica de color negro, durante los primeros 15 días después de la siembra (dds), para un total de ocho tratamientos con tres repeticiones, que corresponden a 24 unidades experimentales (UE). Cada UE estuvo compuesta por 30 semillas.

Las variables de respuesta durante la emergencia de las plántulas fueron la velocidad media de emergencia (VME), tiempo medio de emergencia (TME) y el porcentaje de emergencia (PE), para lo cual, se tuvo en cuenta el número de días gastados por cada semilla para emerger (Tabla 1). A los 60 dds, se evaluó longitud de raíz principal y altura de plántulas.

Se efectuó un análisis de varianza (Anova) y se aplicó la prueba de comparación múltiple de promedios de Tukey, con una confiabilidad del $95 \%$. Se realizó la prueba de correlaciones de Pearson. Para el procesamiento de los datos, se manejó el paquete estadístico SAS v. 8.1e (Cary, N.C).

\section{RESULTADOS Y DISCUSIÓN}

El porcentaje de emergencia presentó diferencias significativas entre tratamientos $(P \leq 0,01)$. Las semillas sometidas a $360 \mathrm{~h}$ de estratificación a $4^{\circ} \mathrm{C}$ y con plástico y a $720 \mathrm{~h}$ con plástico durante $15 \mathrm{dds}$ fueron las únicas que emergieron. $\mathrm{El}$ tratamiento de $360 \mathrm{~h}$ con plástico registró la respuesta más favorable, con un porcentaje de emergencia cercano al $90 \%$, valor muy superior a los demás tratamientos, que permitieron la emergencia de las plántulas (Tabla 2). Respecto al factor tiempo, la estratificación durante $360 \mathrm{~h}$ generó el mayor PE $(P \leq 0,01)$, con 0 y 1440 h, no se indujo emergencia de plántulas (Tabla 3). El efecto de la cobertura fue significativo $(P \leq 0,01)$, hubo mayor PE con la utilización de plástico durante 15dds (Tabla 4). Los porcentajes de mayor valor alcanzados en este experimento fueron superiores a los reportados por Delanoy et al. (2006), al someter semillas de P. mollissima, P. tricuspis Mast. y $P$. nov sp. a diferentes tratamientos pre-germinativos.

Tabla 1. Fórmulas para el cálculo de las variables de emergencia, medidas en la evaluación del efecto de la estratificación fría y la cobertura plástica, en semillas sobre la obtención de plántulas de gulupa.

\begin{tabular}{|c|c|c|}
\hline VARIABLE & ECUACIÓN & UNIDADES \\
\hline $\begin{array}{c}\text { Velocidad media de } \\
\text { emergencia }\end{array}$ & VME $=\sum\left(\frac{\mathrm{n}_{\mathrm{i}}}{\mathrm{t}_{\mathrm{i}}}\right)$ & $\begin{array}{c}\text { Semillas germinadas } \\
\text { /día }\end{array}$ \\
\hline Tiempo medio de emergencia & $\mathrm{TME}=\frac{\mathrm{N} *\left(\mathrm{~A}_{1}+\mathrm{A}_{2}+\mathrm{A}_{\mathrm{X}}\right)}{\left(\mathrm{A}_{1} * \mathrm{~T}_{1}+\mathrm{A}_{2} * \mathrm{~T}_{2}+\mathrm{A}_{\mathrm{X}} * \mathrm{~T}_{\mathrm{X}}\right)}$ & Días \\
\hline Porcentaje de emergencia & $\mathrm{PE}=\left(\frac{\mathrm{N}}{\mathrm{N}_{\mathrm{S}}}\right) * 100$ & $\%$ \\
\hline
\end{tabular}

$\mathrm{n}_{\mathrm{i}}=$ Número de semillas emergidas en el i-ésimo día.

$t_{i}=$ Tiempo en días, para la emergencia en el i-ésimo día.

$\mathrm{N}=$ Número de semillas emergidas.

$A_{1}, A_{2}, \ldots, A_{x}:$ Número de semillas emergidas en el día 1, en el día 2, y en el día $x$.

$T_{1}, T_{2}, \ldots, T_{x}^{x}$ : Número de días entre la siembra y el primer día 1 de emergencia, entre el día 2 y entre el día $x$. 
Tabla 2. Efecto de la estratificación fría y la cobertura plástica en semillas de gulupa, sobre el porcentaje de emergencia (PE), Velocidad media de emergencia (VME), tiempo medio de emergencia (TME), longitud de la raíz principal y altura de las plántulas.

\begin{tabular}{|c|c|c|c|c|c|}
\hline TRATAMIENTO & PE (\%) & $\begin{array}{c}\text { TME } \\
\text { (Días) }\end{array}$ & $\begin{array}{c}\text { VME (Semillas } \\
\text { emergidas/día) }\end{array}$ & $\begin{array}{c}\text { ALTURA } \\
(\mathrm{cm})\end{array}$ & $\begin{array}{c}\text { LONGITUD } \\
\text { DE RAíz } \\
\text { PRINCIPAL }(\mathrm{cm})\end{array}$ \\
\hline 0 h frío y plástico & $0,0 \mathrm{c}$ & $0,0 \mathrm{a}$ & $0,0 \mathrm{c}$ & $0,0 \mathrm{c}$ & $0,0 \mathrm{~b}$ \\
\hline $360 \mathrm{~h}$ frío y plástico & $88,9 \mathrm{a}$ & $38,6 \mathrm{a}$ & $0,69 \mathrm{a}$ & $2,88 \mathrm{a}$ & $8,1 \mathrm{a}$ \\
\hline $720 \mathrm{~h}$ frío y plástico & $33,3 \mathrm{~b}$ & $40,3 \mathrm{a}$ & $0,24 \mathrm{~b}$ & $2,75 \mathrm{a}$ & $11,0 \mathrm{a}$ \\
\hline $1440 \mathrm{~h}$ frío y plástico & $0,0 \mathrm{c}$ & $0,0 \mathrm{a}$ & $0,0 \mathrm{c}$ & $0,0 \mathrm{c}$ & $0,0 \mathrm{~b}$ \\
\hline $0 \mathrm{~h}$ frío y plástico & $0,0 \mathrm{c}$ & $0,0 \mathrm{a}$ & $0,0 \mathrm{c}$ & $0,0 \mathrm{c}$ & $0,0 \mathrm{~b}$ \\
\hline $360 \mathrm{~h}$ frío y sin plástico & $11,1 \mathrm{c}$ & $32,7 \mathrm{a}$ & $0,07 \mathrm{c}$ & $1,36 \mathrm{~b}$ & $6,23 \mathrm{a}$ \\
\hline $720 \mathrm{~h}$ frío y sin plástico & $0,0 \mathrm{c}$ & $0,0 \mathrm{a}$ & $0,0 \mathrm{c}$ & $0,0 \mathrm{c}$ & $0,0 \mathrm{~b}$ \\
\hline $1440 \mathrm{~h}$ frío y sin plástico & $0,0 \mathrm{c}$ & $0,0 \mathrm{a}$ & $0,0 \mathrm{c}$ & $0,0 \mathrm{c}$ & $0,0 \mathrm{~b}$ \\
\hline
\end{tabular}

Promedios con letras iguales en la misma columna no presentan diferencias estadísticas, según la prueba de Tukey $(P \leq 0,05)$.

Tabla 3. Efecto del tiempo de estratificación fría en semillas de gulupa, sobre el porcentaje de emergencia (PE), Velocidad media de emergencia (VME), tiempo medio de emergencia (TME), longitud de la raíz principal y altura de las plántulas.

\begin{tabular}{|c|c|c|c|c|c|}
\hline $\begin{array}{c}\text { HORAS FRÍO } \\
\left(4^{\circ} \mathrm{C}\right)\end{array}$ & PE (\%) & TME (Días) & $\begin{array}{c}\text { VME (Semillas } \\
\text { emergidas/día) }\end{array}$ & ALTURA (cm) & $\begin{array}{c}\text { LONGITUD } \\
\text { DE RAíz } \\
\text { PRINCIPAL } \\
(\mathrm{cm})\end{array}$ \\
\hline 0 & $0,0 \mathrm{c}$ & $0,0 \mathrm{~b}$ & $0,0 \mathrm{~b}$ & $0,0 \mathrm{~b}$ & $0,0 \mathrm{~b}$ \\
\hline 360 & $49,9 \mathrm{a}$ & $35,6 \mathrm{a}$ & $0,38 \mathrm{a}$ & $2,1 \mathrm{a}$ & $7,2 \mathrm{a}$ \\
\hline 720 & $16,6 \mathrm{~b}$ & $20,13 \mathrm{a}$ & $0,12 \mathrm{a}$ & $1,4 \mathrm{a}$ & $5,5 \mathrm{a}$ \\
\hline 1440 & $0,0 \mathrm{c}$ & $0,0 \mathrm{~b}$ & $0,0 \mathrm{~b}$ & $0,0 \mathrm{~b}$ & $0,0 \mathrm{~b}$ \\
\hline
\end{tabular}

Promedios con letras iguales en la misma columna no presentan diferencias estadísticas, según la prueba de Tukey $(P \leq 0,05)$.

Tabla 4. Efecto de la cobertura sobre el porcentaje de emergencia (PE), Velocidad media de emergencia (VME), tiempo medio de emergencia (TME), longitud de la raíz principal y altura de las plántulas de gulupa.

\begin{tabular}{|c|c|c|c|c|c|}
\hline COBERTURA & PE (\%) & $\begin{array}{c}\text { TME } \\
\text { (Días) }\end{array}$ & $\begin{array}{c}\text { VME (Semillas } \\
\text { emergidas/día) }\end{array}$ & $\begin{array}{c}\text { ALTURA } \\
(\mathrm{cm})\end{array}$ & $\begin{array}{c}\text { LONGITUD } \\
\text { DE RAíz } \\
\text { PRINCIPAL (cm) }\end{array}$ \\
\hline Plástico & $30,5 \mathrm{a}$ & $19,7 \mathrm{a}$ & $0,23 \mathrm{a}$ & $1,39 \mathrm{a}$ & $4,77 \mathrm{a}$ \\
\hline Sin plástico & $2,8 \mathrm{~b}$ & $8,2 \mathrm{~b}$ & $0,01 \mathrm{~b}$ & $0,34 \mathrm{~b}$ & $1,55 \mathrm{~b}$ \\
\hline
\end{tabular}

Promedios con letras iguales en la misma columna no presentan diferencias estadísticas, según la prueba de Tukey $(P \leq 0,05)$. 
La germinación consiste en la emergencia y el desarrollo del embrión y de todas las estructuras esenciales para producir una plántula normal, bajo condiciones favorables (AOSA, 2000); sin embargo, las semillas de Passiflora spp. tienen dormancia exógena, producto de una posible combinación entre latencia química y mecánica (Ellis et al. 1985; Bewley $\mathcal{E}$ Black, 1994, Hartmann et al. 1997; Schmidt, 2000; Delanoy et al. 2006).

La temperatura es considerada uno de los factores mas importantes en la germinación de la mayoría de las especies vegetales (Probert, 2000). Los resultados sugieren que la estratificación en frío actúa como un promotor de la germinación y se puede deber a adaptaciones al ambiente natural, en los cuales, se originó la especie (Manjkhola et al. 2003).

Teniendo en cuenta que la gulupa procede del sur de América, las semillas pueden experimentar estratificación fría en su ambiente natural, resultando en una mayor germinación, en las siguientes temporadas. Además, se ha reportado que la estratificación fría aumenta la síntesis de giberelinas y disminuye la concentración de inhibidores de germinación en las semillas, como el ácido abscísico (ABA) (Kulkarni et al. 2006). La exposición al frío degrada el ABA presente en las semillas a ácido faséico y dihidroxifaséico, que son compuestos inactivos, lo que, a su vez, aumenta la relación $\mathrm{GA}_{3} / \mathrm{ABA}$, para que ocurra la germinación (Davies, 2004). Rossetto et al. (2000) encontraron que las semillas de $P$. alata sin arilo y sometidos a remojo previo con $\mathrm{GA}_{3}$ mostraron un incremento en el porcentaje de germinación. Según Passos et al. (2004), la aplicación de $1000 \mathrm{mg} \mathrm{L}^{-1}$ de $\mathrm{GA}_{3}$ con luz fue efectiva para romper la latencia de las semillas de $P$. nitida Kunth, con una germinación del $86 \%$.

A pesar de encontrarse a la misma temperatura, la germinación disminuyó con el tiempo de estratificación de las semillas de gulupa, lo que sugiere que el poder germinativo de esta especie no se mantiene por un periodo muy largo. La pérdida de poder germinativo se ha reportado para otras pasifloras, como $P$. edulis, mientras $P$. alata no presenta problemas con el almacenamiento (Osipi \& Nakagawa, 2005; Alves et al. 2006).

Por otro lado, las condiciones de luz desempeñan un papel crucial en la germinación, siendo más importante la calidad que la cantidad de luz (Casal $\&$ Smith, 1989). No es muy usual que la germinación se vea influenciada negativamente por la luz; sin embargo esto ocurre en especies de diferentes familias y entre ellas las pasifloras (Kulkarni et al. 2006).

Las semillas de $P$. incarnata L. son fotoblásticas negativas y altamente exigentes en cuanto a requerimientos de calor. El mayor porcentaje (90\%) de germinación, se logra a $35^{\circ} \mathrm{C}$; a temperaturas más bajas, este valor disminuye drásticamente y se reduce entre un $50 \%$ y $15 \%$ a 30 y $25^{\circ} \mathrm{C}$, respectivamente (Benvenuti et al. 2001). Otra pasiflora con este comportamiento es $P$. cincinnata Mast., en la cual, la luz parece ejercer un efecto inhibitorio sobre la germinación, ya que las semillas fueron germinadas a temperaturas alternas $20-30^{\circ} \mathrm{C}$; las semillas que brotan en la oscuridad muestran el máximo de germinación en la oscuridad, registrando 14,4\% de germinación, mientras que bajo las mismas condiciones, pero sometiendo las semillas a la luz, el porcentaje máximo de germinación fue de 3,2\% (Zucareli et al. 2009). Los resultados para estas especies y, especialmente, para P. edulis, concuerdan con las las reglas para el análisis de semillas (Brasil, 2009), en las que se indica que el test de geminación, se debe hacer en ausencia de luz.

Los resultados encontrados sugieren que las semillas de gulupa son fotoblásticas negativas con requerimiento de estratificación fría, no obstante, se desconocen las reacciones que regulan el proceso. El fitocromo puede controlar la germinación de las semillas fotoblásticas negativas, mediante la respuesta a las condiciones de alta radiación (Kulkarni et al. 2006). En investigaciones con semillas de mutantes de Arabidopsis incubadas en la oscuridad, se encontró que la germinación era controlada, principalmente, por el fitocromo B (Von Arnim $\varepsilon$ Deng, 1996). Takaki (2001) sugirió que las formas del fitocromo en el control de la germinación podrían reemplazar el término fotoblasticismo; en el caso de las semillas fotoblásticas negativas, su germinación está controlada por el fitocromo A, bajo condiciones de alta radiación. Por el contrario, Benvenuti et al. (2001) sugieren que la influencia del fitocromo es muy débil o no se da en semillas de $P$. incarnata, basados en el hecho de que la exposición de las semillas a repetidas irradiaciones con luz roja y roja lejana no produjeron diferencias en el porcentaje de germinación.

El tiempo de emergencia no arrojó diferencias estadísticas entre los tratamientos que indujeron germinación. El menor tiempo gastado para la germinación, se consiguió con 360h sin plástico y el mayor tiempo con $720 \mathrm{~h}$ y plástico, que demoró 40 días (Tabla 2). El factor tiempo de estratificación fue estadísticamente diferente, hubo mayor TME con 360h (Tabla 3). Con la utilización de cobertura plástica, el TME también fue mayor (Tabla 4). Esto ocurrió debido a la relación directamente proporcional entre el TME y el PE, ya que el mayor número de plántulas emergidas se produjo en un mayor periodo de tiempo.

Las plántulas de gulupa, en el momento de la emergencia, alargaron el hipocótilo y elevaron los cotiledones sobre el sustrato de germinación, por lo tanto, presentaron una germinación epigea (Hartmann $\mathcal{E}$ Kester, 2000).

Solo tres combinaciones, de las horas frío y la presencia de plástico negro, favorecieron la germinación de las semillas de gulupa. Se ha comprobado que la luz no influye de forma aislada, son combinaciones determinadas entre temperatura y luz las 
que favorecen la germinación (Toole, 1976). En semillas de $P$. incarnata, el tiempo medio de germinación fue inversamente proporcional a las temperaturas, independientemente, de las condiciones de luz (Benvenuti et al. 2001).

Cuanto mayor es el tiempo entre la germinación y la emergencia, mayor es la probabilidad que la semilla pierda viabilidad (Arjona et al. 1998), por lo anterior, para asegurar la supervivencia de la plántula, la germinación de las semillas de gulupa debe ser rápida, para permitir que los procesos metabólicos posteriores se lleven a cabo, de forma normal. El mayor número de semillas emergidas por día, se logró con 360h y, la utilización de plástico, en los primeros 15dds, con diferencias altamente significativas, respecto a los demás tratamientos, que permitieron la emergencia de las plántulas (Tabla 2). Con $360 \mathrm{~h}$, se obtuvo mayor VME $(P \leq 0,01)$ respecto a los demás tiempos de estratificación (Tabla 3) y el empleo de cobertura plástica durante 15 dds generó mayor VME, en relación con las semillas, a las cuales, no se les colocó plástico (Tabla 4).

En $P$. incarnata, los efectos negativos de la luz sobre la germinación de las semillas ocurrieron en las primeras 24 y 36 horas de incubación, como función de la temperatura (35 y $30^{\circ} \mathrm{C}$ ). El espectro del rojo y el rojo lejano inhibió la germinación de mayor forma que el blanco o el azul, a $30^{\circ} \mathrm{C}$, pero a $35^{\circ} \mathrm{C}$, no se observaron diferencias significativas en la tasa de germinación (Benvenuti et al. 2001).

Según la tabla 2, entre los tratamientos que permitieron la emergencia no se presentó diferencias estadísticas para la longitud de la raíz; sin embargo, la raíz principal más extensa, se logró con $720 \mathrm{~h}$ y, con plástico, en los primeros $15 d d s$. El análisis factorial indicó que $360 \mathrm{~h}$ de estratificación favorecieron la longitud de raíz (Tabla 3), al igual que la cobertura plástica (Tabla 4). El tratamiento con la más baja VME de igual forma exhibió la menor longitud de raíz principal, ya que las plántulas que emergen primero inician también más temprano a desarrollar su sistema radicular, respecto a las que emergen después. El sistema radicular tiene importantes funciones físicas y fisiológicas desde el inicio de la germinación y emergencia, hasta el crecimiento y desarrollo del transplante. El tamaño, la morfología y la arquitectura pueden ejercer un control sobre el tamaño relativo y ritmo de crecimiento del tallo (Leskovar, 2001).

Se registraron diferencias altamente significativas en la altura de plántulas de gulupa; se obtuvo mayor altura con aquellas semillas sometidas a 360h, con plástico, en los primeros 15 dds (Tabla 2), según el análisis estadístico; estos factores por separado también generaron la mayor altura (Tablas 3 y 4). Se observa que las plántulas que tienen una menor longitud de raíz principal desarrollan, igualmente, una menor parte aérea (datos no mostrados). El estrés ambiental y biológico originado en la rizósfera puede ser expresado en el tallo, afectando la partición de biomasa, el desarrollo vegetativo y, finalmente, la productividad del transplante (Leskovar, 2001).

Buitrago-Rueda et al. (2004) obtuvieron una mayor altura de plantas en semillas de Manilkara zapota sembradas sin luz y, al igual que en las semillas de gulupa, puede estar asociado a un incremento en la concentración de giberelinas, regulador de crecimiento que promueve el alargamiento de las regiones subapicales (Weaver, 1976) y, por ende, el desarrollo de tallos más largos (Buitrago-Rueda et al. 2004). El posible aumento de las giberelinas en el presente estudio, se pudo causar previo a la emergencia, momento en el que todavía existía la cobertura plástica, lo que supone una relación entre el incremento de la humedad relativa, la temperatura y la ausencia de luz, con el aumento de la concentración de $\mathrm{GA}_{3}$.

Según el análisis de correlaciones de Pearson, se puede observar que todas las correlaciones fueron positivas y significativas. Se encontró que al haber un mayor PE, se logró mayor velocidad y tiempo de emergencia, altura de planta y longitud de raíz principal (Tabla 5). Esto ratifica el hecho

Tabla 5. Correlaciones de Pearson para las variables evaluadas, efecto de la estratificación fría y la cobertura plástica, en semillas sobre la obtención de plántulas de gulupa.

\begin{tabular}{|c|c|c|c|c|c|}
\hline VARIABLES & PE & TME & VME & ALTURA & $\begin{array}{c}\text { LONGITUD DE RAÍZ } \\
\text { PRINCIPAL }\end{array}$ \\
\hline PE & 1 & $0,79^{* *}$ & $0,99^{* *}$ & $0,86^{* *}$ & $0,76^{*}$ \\
\hline TME & & 1 & $0,77^{*}$ & $0,97^{* *}$ & $0,97^{* *}$ \\
\hline VME & & & 1 & $0,85^{* *}$ & $0,71^{*}$ \\
\hline Altura & & & & 1 & $0,97^{* *}$ \\
\hline Longitud de raíz principal & & & & & 1 \\
\hline
\end{tabular}

** Significancia al 1\%; * Significancia al $5 \%$, según prueba de Pearson. 
que la estratificación de semillas de gulupa durante 360h y la puesta de plástico negro durante la germinación favorece un mayor PE y, a su vez, permite la obtención de plántulas de mejor calidad.

Finalmente, se considera que la estratificación durante 360 horas a $4^{\circ} \mathrm{C}$ y la utilización de cobertura plástica negra durante los primeros $15 \mathrm{dds}$ son necesarias para activar la germinación de semillas de gulupa y conseguir un alto porcentaje de germinación. Además, la altura de las plántulas de gulupa guarda una relación directa con la velocidad media y el tiempo medio de emergencia.

Conflictos de interés: La investigación y el manuscrito, se realizaron con la colaboración de todos los autores, quienes declaramos que no existe ningún conflicto de interés que ponga en riesgo la validez de los resultados presentados.

\section{BIBLIOGRAFÍA}

1. ALEXANDRE, R.S.; JÚNIOR, A.W.; NEGREIROS, J.R.S.; PARIZZOTTO, A.; BRUCKNER, C.H. 2004. Germinação de sementes de genótipos de maracujazeiro. Pesquisa Agropec. Bras. 39:1239-1245.

2. ALVES, C.Z.; de SÁ, M.E.; CORREA, L.; BINOTTI, F. 2006. Efeito da temperatura de armazenamento e de fitorreguladores na germinacao de sementes de maracujá doce e desenvolvimento iniial e mudas. Acta Sci. Agron. (Brasil). 28(3):441-448.

3. ARJONA, H.; GUERRERO, A.; PRIETO, C. 1998. Estudios de osmoiniciación de semillas de cebolla de bulbo Allium cepa L. Agronomía Colombiana. 15(2):143-152.

4. AOSA. ASSOCIATION OF OFFICIAL SEED ANALYSTS. 2000. Rules for testing seeds. Bozeman, Mont. 126p.

5. BENVENUTI, S.; SIMONELLI, G.; MACCHIA, M. 2001. Elevated temperature and darkness improve germination in Passiflora incarnata L. seed. Seed Science and Technology (Suiza). 29:533-541.

6. BEWLEY, J.D.; BLACK, M. 1994. Seeds: physiology of development and germination. Plenum Press (New York). 445p.

7. BORGES, E.E.L.; RENA, A.B. 1993. Germinação de sementes. En: AGUIAR, I.B.; PINÃ-RODRIGUES, F.C.M.; FIGLIOGLIA, M.B. Sementes florestais tropicais. Brasília, DF: Abrates. p. 83-135.
8. BRASIL. 2009. Regras para Análise de Sementes. Ministério da Agricultura e Reforma Agrária. Secretaria Nacional de Defesa Agropecuária. Departamento Nacional de Produção Vegetal. Coordenação de Laboratório Vegetal. Brasília, DF, (Brasil). 395p.

9. BUITRAGO-RUEDA, N.; RAMÍREZ-VILLALOBOS, M.; GÓMEZ-DEGRAVES, A.; RIVERO-MALDONADO, G.; PEROZO-BRAVO, A. 2004. Efecto del almacenamiento de las semillas y la condición de luz postsiembra sobre la germinación y algunas características morfológicas de plantas de níspero (Manilkara zapota (Van Royen) (Jacq) Gill) a nivel de vivero. Rev. Fac. Agron. (Venezuela) (LUZ). 21:343-352.

10. CALZADA, B.J. 1970. 143 frutales nativas. El maracuyá. Universidad Nacional Agraria, La Molina, Lima, Perú. p.320-322.

11. CAMACARO, N.; PÉREZ, D.; de MIRANDA, G.; CARVAJAL, R.; ESPINOZA, M. 2005. Métodos para la ruptura de latencia de semillas de parchita (Passiflora edulis Sims. f. flavicarpa). Fac. Agron. U. Central de Venezuela, Maracay, Estado Aragua. Instituto Nacional de Investigaciones Agrícolas (INIA). 22p. Disponible desde Internet en: http://www.cedaf.org.do/eventos/ISTH2005/ memoria/Martes/PDF/02.pdf (con acceso 07/01/10).

12. CARLESSO, V.O.; BERBERT, P.A.; DA SILVA, R.F.; DETMANN, E. 2008. Secagem e armazenamento de sementes de maracujá amarelo (Passiflora edulis Sims f. flavicarpa Degener) / Drying and storage of seeds of the yellow passion fruit (Passiflora edulis Sims f. flavicarpa Degener). Rev. Bras. Sementes. 30(2):65-74.

13. CASAL, J.J.; SMITH, H. 1989. The function, action and adaptive significance of phytochrome in light-grown plants. Plant Cell and Environment. (USA). 12:855-862.

14. DAVIES, P. 2004. Plants hormones. First Ed. Kluner Academis Publishers. (United States). 750p.

15. DELANOY, M.; VAN DAMME, P.; SCHELDEMAN, X.; BELTRAN, J. 2006. Germination of Passiflora mollissima (Kunth) L.H.Bailey, P. tricuspis Mast. and P. nov sp. seeds. Scientia Horticulturae (Holanda). 110:198-203.

16. ELLIS, R.H.; HONG, T.D.; ROBERTS, E.H. 1985. Handbook of seed technology for genebanks. International Board for Plant Genetic Resources (IBPGR), Rome, Italy. 163p. 
17. ESTEVES, A.C.; ZUCARELI, V.; MISCHAN, M.; FERREIRA, G. 2009. Combinações entre $\mathrm{GA}_{4+7}+\mathrm{N}$-(fenilmetil)aminopurina e ethephon na germinação de sementes de Passiflora cincinnata Mast. Rev. Bras. Sementes. 31(1):216-223.

18. HARTMANN, H.T.; KESTER, D.E. 2002. Propagación de plantas: principios y prácticas. $7^{\mathrm{a}}$ ed. Compañía Editorial Continental. México, D.F. 896p.

19. HARTMANN, H.T.; KESTER, D.E. 2000. Propagación de Plantas. Principios y Prácticas. $8^{a}$ Reimpresión. Editorial Continental. (México). 760p.

20. HARTMANN, H.T.; KESTER, D.E.; DAVIES, F.T. 1997. Plant Propagation: Principles and Practices, Prentice Hall International (New Jersey). 647p.

21. HECHENLEITNER, V.P.; GARDNER, M.F.; THOMAS, P.I.; ECHEVERRÍA, C.; ESCOBAR, B.; BROWNLESS, P.; MARTÍNEZ, C. 2005. Plantas Amenazadas del CentroSur de Chile. Distribución, Conservación y Propagación. $1^{\text {a }}$ Ed. Universidad Austral de Chile y Real Jardín Botánico de Edimburgo. 188p.

22. KULKARNI, M.G.; SPARG, S.G.; VAN STANDEN, J. 2006. Dark conditioning, cold stratification and a smoke-derived compound enhance the germination of Eucomis autumnalis subsp. autumnalis sedes. South African J. Botany. 72:157-162.

23. LA ROSA, A.M. 1984. The biology and ecology of Passiflora mollissima in Hawaii. Cooperative National Park Resources Studies Unit. Tech. Report. 50p.

24. LESKOVAR, D.I. 2001. Producción y Ecofisiología del transplante hortícola. Curso dado en Buenavista, Saltillo, Coahuila. Disponible desde internet en: ww.uaaan. mx/academic/Horticultura/Memhort01/ Curso.pdf. (con acceso 07/02/10).

25. MADUEÑO-MOLINA, A.; GARCÍA-PAREDES, D.; MARTÍNEZ-HERNÁNDEZ, J.; RUBIO-TORRES, C.; NAVARRETE-VALENCIA, A.; BOJÓRQUEZ-SERRANO, J. 2006. Germinación de semilla de frijolillo, Rhynchosia minima (L.) Dc., luego de someterla a tratamientos pregerminativos. Bioagro. (Venezuela). 18(2):101-105.

26. MANJKHOLA, S.; DHAR, U.; RAWAL, R.S. 2003. Treatments to improve seed germination of Arnebia benthamii: an endangered medicinal herb of high altitude Himalaya. Seed Science and Technology. (Suiza). 31:571-577.
27. MORTON, J.F. 1987. Fruits of warm climates. Creative Resources Systems, Inc. Winterville, NC. (USA). 505p.

28. NAKASONE, H.; PAULL, R.E. 1998. Tropical fruits. CAB Internacional, Wallington, U.K. p.270-291.

29. OSIPI, E.; NAKAGAWA, J. 2005. Efeito da temperatura na avalicao da qualidade fisiológica de sementes do maracujá-doce (Passiflora alata Dryander). Rev. Bras. Fruticultura. 26(2):53-63.

30. PASSOS, I.R.S.; MATOS, G.V.C.; MELETTI, L.M.M.; SCOTT, M.D.S.; BERNACCI, L.C.; VIEIRAS, M.A.R. 2004. Utilização do ácido giberélico para a quebra de dormência de sementes de Passiflora nitida Kunth germinadas in vitro. Rev. Brás. Fruticultura. 26:380-381.

31. PEREIRA, T.S.; ANDRADE, A.C.S. 1994. Germinação de Psidium guajava L. e Passiflora edulis Sims - Efeito da temperatura, substrato e morfologia do desenvolvimento pós-seminal. Rev. Bras. de Sementes. 16(1):58-62.

32. PINZÓN, I.M.; FISCHER, G.; CORREDOR, G. 2007. Determinación de los estados de madurez del fruto de la gulupa (Passiflora edulis Sims.). Agronomía Colombiana. 25(1):83-95.

33. PROBERT, R.J. 2000. The role of temperature in the regulation of seed dormancy and germination. In: Fenner, M. (Ed.), Seeds: The Ecology of Regeneration in Plant Communities, $2^{\text {nd }}$ ed. CABI Publishing, Wallingford, UK, p.261-292.

34. ROLSTON, M.P. 1978. Water impermeable seed dormancy. Bot. Rev. (USA). 44:365-396.

35. ROSSETTO, C.A.V.; CONEGLIAN, R.C.C.; NAKAGAWA, J.; SHIMIZU, M.K.; MARIN, V.A. 2000. Germinação de sementes de maracujá-doce (Passiflora alata Dryand) em função de tratamento pré-germinativo. Revista Brasileira de Sementes. 22(1): 247-252.

36. SCHMIDT, L. 2000. Guide to Handling of Tropical and Subtropical Forest Seed, Danida Forest Seed Centre, Humlebaek, (Denmark). 511p.

37. TAKAKI, M. 2001. New proposal of classification of seeds based on forms of phytochrome instead of photoblastism. Rev. Bras. Fisiol. Vegetal. 13:104-108.

38. TOOLE, V.K. 1976. Effects of light, temperature and their interaction on the germination of seeds. Seed Science and Technology, (Zürich).1(2):339-396. 
39. VON ARNIM, A.; DENG, X. 1996. Light control of seedling development. Annu. Rev. Plant Physiol. Plant Molecular Biology. (USA). 47:215-244.

40. WEAVER, R. 1976. Reguladores del crecimiento de las plantas en la agricultura. Editorial Trillas. (México). 97p.

41. WEHTJE, G.; REED, R.B.; DUTE, R.R. 1985. Reproductive biology and herbicidal sensitivity of maypop passionflower (Passiflora incarnata), Weed Sci. (USA). 33:484-490.

42. WENKAM, N.S. 1990. Food of Hawaii and the Pacific Basin, Fruits and Fruit Products: Raw, Processed, and Prepared. Vol. 4: Composition. Hawaii Agricultural Experiment Station Research and Extension Series 110, 96p.
43. ZUCARELI, V.; FERREIRA, G.; ESTEVES, A.C.; PINHEIRO, F. 2009. Fotoperíodo, temperatura e reguladores vegetais na germinação de sementes de Passiflora cincinnata Mast. Revista Brasileira de Sementes. 31(3):106-114.

Recibido: Octubre 15 de 2009

Aceptado: Octubre 17 de 2010 